\title{
Avaliação de pacientes com transtorno afetivo bipolar baseada na Escala de Disfunções Cognitivas no Transtorno Bipolar (COBRA)
}

\author{
Assessment of patients with bipolar affective disorder based on the Cognitive Disorders in \\ Bipolar Disorder Scale (COBRA)
}
Evaluación de pacientes con trastorno afectivo bipolar basada en la Escala de Trastornos Cognitivos en Trastorno Bipolar (COBRA)

Mateus Teixeira de Moura ${ }^{1,2 *}$, Gabriela Lopes Martins ${ }^{3}$, Ana Luísa Lamounier Costa ${ }^{1,2}$, Lair da Silva Gonçalves ${ }^{1,2}$.

\section{RESUMO}

Objetivo: Avaliar o desempenho neurocognitivo de pacientes com transtorno afetivo bipolar (TAB), acompanhados em ambulatório especializado, utilizando a escala de disfunções cognitivas no transtorno bipolar (Cognitive Complaints in Bipolar Disorder Rating Assessment - COBRA). Métodos: Realizou-se um estudo observacional, analítico e transversal, no qual foram avaliados 18 pacientes com TAB acompanhados em ambulatório especializado, com idade entre 18 e 60 anos, além de 15 indivíduos do grupo controle, durante entrevista médica. Todos os participantes foram submetidos à avaliação pela escala COBRA e, além disso, dados clínicos e demográficos foram coletados. Resultados: Os pacientes com TAB, cuja idade mediana foi de 39 anos, apresentaram pior desempenho cognitivo, demonstrado pela maior pontuação na COBRA $(20,6$ $\pm 9,7)$, em relação aos controles $(5,3 \pm 2,3)$. Além disso, também foi observado elevado tempo de afastamento do trabalho dentre os pacientes com o transtorno, com média de 5,7 $\pm 2,4$ anos, e apenas quatro (22,2\%) relataram estar trabalhando atualmente. Conclusão: A escala COBRA é um instrumento viável de ser aplicado na rotina ambulatorial, a qual pode ser uma forma complementar acompanhamento, com vistas a minimizar as interferências que o prejuízo cognitivo causa nas áreas social e ocupacional dos pacientes com TAB.

Palavras-chave: Transtorno bipolar, Cognição, Saúde mental.

\begin{abstract}
Objective: Evaluate the neurocognitive performance of patients with bipolar affective disorder (BD), followed up in a specialized outpatient clinic, using the Cognitive Complaints in Bipolar Disorder Rating Assessment (COBRA). Methods: An observational, analytical and cross-sectional study was carried out enrolling 18 patients with BD, aged between 18 and 60 years, in addition to 15 individuals in the control group, evaluated during a medical interview. All participants were submitted to the assessment using the COBRA scale and clinical and demographic data were also collected. Results: Patients with BD, whose median age was 39 years, had worse cognitive performance, demonstrated by the higher score on COBRA (20.6 \pm 9.7$)$, compared to controls $(5.3 \pm 2.3)$. Moreover, a long time off work was also observed among patients with the disorder, with an average of $5.7 \pm 2.4$ years, and only four (22.2\%) reported being currently working. Conclusion: COBRA scale is a viable instrument to be applied in the outpatient routine, which can be a complementary form of monitoring, in order to minimize the interferences that the cognitive impairment causes in the social and occupational areas of patients with BD.
\end{abstract}

Keywords: Bipolar disorder, Cognition, Mental health.

1 Secretaria de Estado de Saúde do Distrito Federal, Brasília - DF. *E-mail: mateusteixeira141@gmail.com

2 Hospital São Vicente de Paulo, Brasília - DF.

3 Universidade Federal de Minas Gerais (UFMG), Belo Horizonte - MG. 


\section{RESUMEN}

Objetivo: Evaluar el desempeño neurocognitivo de pacientes con trastorno afectivo bipolar (TAB), seguido de una clínica ambulatoria especializada, utilizando una escala de trastornos cognitivos en el trastorno bipolar (Cognitive Complaints in Bipolar Disorder Rating Assessment - COBRA). Métodos: Se realizó un estudio observacional, analítico y transversal, sin que se siguiera a 18 pacientes con TAB en consulta externa especializada, con edades comprendidas entre 18 y 60 años, además de 15 obligatoriamente del grupo control, durante la consulta médica. entrevista, fueron evaluados. Todos los participantes fueron recolectados para su evaluación utilizando la escala COBRA y, además, se recolectaron datos clínicos y demográficos. Resultados: Pacientes con TAB, mediana de edad de 39 años, peor desempeño cognitivo, marcado por la puntuación más alta en COBRA $(20,6 \pm 9,7)$, en comparación con los controles $(5,3 \pm 2,3)$. Además, también se observó una baja laboral prolongada entre los pacientes con el trastorno, con una media de 5,7 $\pm 2,4$ años, y solo cuatro $(22,2 \%)$ informaron estar trabajando actualmente. Conclusión: La escala COBRA es un instrumento viable para ser aplicado en la rutina ambulatoria, que puede ser una forma complementaria de seguimiento, con el fin de minimizar la interferencia que ocasiona el deterioro cognitivo en el ámbito social y laboral de los pacientes con TAB.

Palabras clave: Trastorno bipolar, Cognición, Salud mental.

\section{INTRODUÇÃO}

O transtorno afetivo bipolar (TAB) é um dos mais graves tipos de transtorno mental, caracterizando-se pela alternância de episódios de humor (mania/hipomania e depressão), os quais apresentam grande variabilidade em intensidade, duração e frequência (CARVALHO AF, et al., 2020; GOODWIN FK e JAMISON KR, 2007).

O TAB é um importante problema de saúde pública no campo da saúde mental, com prevalência estimada na população dos Estados Unidos de aproximadamente 3,9\%, variando entre 1,5 e 6\%. No Brasil, após reanálise dos dados do Estudo de Área de Captação Epidemiológica, estimou-se, em subgrupos de TAB que incluíam pacientes com hipomania subsindrômica e sintomas maníacos, prevalência de $8,3 \%$ ao longo da vida (COSTA AMN, 2008).

Há também uma significativa associação entre aumento do risco da mortalidade em pacientes com TAB, com dados apontando que $25 \%$ dos pacientes tentam suicídio durante a vida e $11 \%$ acabam cometendo autoextermínio (HILTY DM, et al., 1999; JAMES SL, et al., 2018; VIGO D, et al., 2016).

A mania é caracterizada por humor anormal e persistentemente elevado, expansivo ou irritável. Pode haver redução do sono, hipersexualidade e comportamentos de risco, assim como alterações do pensamento, sentimento de grandiosidade, e hiperatividade motora. Há, ainda, possibilidade de associação com sintomas psicóticos. A hipomania, por sua vez, é um estado que se assemelha à mania, mas com menor duração e sintomas mais brandos, sem associação com psicose. Já a depressão é um estado psíquico onde há uma sensação de infelicidade, com predomínio de pensamentos negativos, apatia, sentimento de culpa, comumente associada a sintomas neurovegetativos - alentecimento psicomotor, diminuição da libido e distúrbios do sono (SADOCK BJ, et al., 2016).

Existem indicadores de gravidade dos episódios nas diferentes fases da doença. Entretanto, sintomas psicóticos tendem a ser um indicador em qualquer fase, mania ou depressão, assim como a alta frequência destes episódios indica maior risco de cronicidade da doença (SHASTRY BS, 2005).

As causas do TAB ainda não são totalmente compreendidas, mas tanto fatores ambientais como genéticos têm influência. Diferentes estudos com famílias e com gêmeos têm demonstrado uma forte contribuição de fatores genéticos herdados para o risco de se desenvolver o transtorno, mas ainda não foi possível identificar nenhum gene isolado capaz de aumentar o risco da doença de forma mendeliana (SQUARCINA L, et al., 2016).

O tratamento do TAB é realizado prioritariamente por via medicamentosa, usando estabilizadores do humor, como o lítio e anticonvulsivantes, bem como alguns antipsicóticos atípicos. A farmacoterapia tem como objetivo a estabilização do quadro, com remissão dos sintomas, retorno à eutimia (estado de equilíbrio do 
humor) e regresso às atividades ocupacionais. O tratamento farmacológico é complexo, porque são utilizados os mesmos medicamentos para mania, hipomania e depressão. Os antidepressivos podem ser usados na fase depressiva em situações excepcionais, mas aumentam a chance de uma virada maníaca (GEDDES JR e MIKLOWITZ DJ, 2013).

Apesar de o tratamento farmacológico ser a intervenção primária e essencial para a manutenção da estabilidade do quadro, abordagens psicoterápicas adicionais ajudam nos episódios depressivos e na prevenção de recaídas. Não existe evidência positiva ou recomendação específica para psicoterapia na fase de mania. Entretanto, a psicoeducação e a terapia comportamental cognitiva tem-se demonstrado como estratégias eficazes no suporte destes pacientes (YATHAM LN, et al., 2018).

Além das alterações de humor, pacientes com TAB também podem apresentar déficits no funcionamento cognitivo, o que tem sido um importante alvo de pesquisa nos últimos anos (KURTZ MM e GERRATY RT, 2009; MANN-WROBEL MC, et al., 2011). Inicialmente, acreditava-se que o prejuízo cognitivo seria mais acentuado nas fases agudas da doença e que os pacientes retornariam ao seu nível funcional após a remissão dos sintomas de humor (DEPP CA, et al., 2016).

Entretanto, há evidências de que as perdas cognitivas podem estar presentes em todas as fases da doença, até mesmo na fase eutímica (BORTOLATO B, et al., 2015). Com isso, as alterações na cognição e o comprometimento funcional passaram a ser consideradas inerentes ao transtorno, uma vez que a maioria dos pacientes não consegue obter recuperação funcional completa, mesmo se tornando assintomáticos com o tratamento (LIMA FM, et al., 2018; STREJILEVICH S, et al., 2013).

$O$ curso da doença é outro fator que tem sido associado à perda cognitiva nos pacientes com $T A B$, com indicativos de correlação positiva da gravidade da doença com o prejuízo cognitivo (ROSA AR, et al., 2013). Nesse sentido, foi proposto que um pior prognóstico poderia estar relacionado à maior número de eventos estressores, como traumas e perdas, maiores ocorrências de episódios de alterações de humor, maior uso de substâncias psicoativas, início de tratamento tardio e pior adesão a este, e maior prevalência de comorbidades clínicas e psiquiátricas (BRASIL, 2016; KAPCZINSKI F, et al., 2008).

Diante desses fatores, houve um aumento pela busca de estratégias de prevenção e tratamento do comprometimento cognitivo em pacientes com TAB, com vistas à melhoria dos aspectos psicossociais, ocupacionais e à qualidade de vida destes indivíduos (MISKOWIAK KW, et al., 2018; ROSA AR, et al., 2013).

Em um estudo que comparou a função neurocognitiva entre pacientes com TAB estáveis, pacientes com esquizofrenia e controles, observou-se que aqueles com TAB apresentaram prejuízos em diversos domínios, embora não em todos, os quais foram menores do que os observados nos pacientes com esquizofrenia. As principais perdas cognitivas observadas foram nos domínios da memória e função executiva (ALTSHULER LL, et al., 2004). Em outro trabalho que avaliou o desempenho cognitivo em indivíduos com TAB, foram observados déficits significativos nos campos da memória de trabalho e atenção (TORRENT C, et al., 2006).

Apesar de sua relevância, a avaliação e o monitoramento da função cognitiva no TAB ainda não são realizados na rotina dos atendimentos clínicos. Recentemente, foi publicado um consenso da Sociedade Internacional de Transtorno Bipolar recomendando a utilização da escala objetiva Screen for Cognitive Impairment in Psychiatry (SCIP) e da escala de disfunções cognitivas no transtorno bipolar (Cognitive Complaints in Bipolar Disorder Rating Assessment - COBRA), um instrumento de avaliação subjetiva (MISKOWIAK KW, et al., 2018).

A SCIP consiste em uma bateria de triagem composta por testes curtos, com duração aproximada de 10 a 15 minutos, que avaliam: memória de trabalho, memória verbal, fluência verbal e velocidade psicomotora (JENSEN JH, et al., 2015). Já a COBRA é uma escala de autoavaliação, que permite verificar dificuldades cognitivas comumente relatadas pelos pacientes e descritas na literatura, tais como problemas relacionados à atenção, concentração, memória, percepção, pensamento, linguagem, movimento e emoção (ROSA AR, et al., 2013). Ela foi traduzida e validada em diferentes idiomas, como inglês, espanhol, francês, japonês e, recentemente, português (Brasil) com validação em pacientes brasileiros (LIMA FM, et al., 2018). 
Diante disso, o objetivo deste estudo foi avaliar o desempenho neurocognitivo de pacientes com TAB acompanhados em um ambulatório de transtornos de humor, utilizando a escala COBRA, a fim de avaliar a sua implementação na rotina de atendimentos.

\section{MÉTODOS}

\section{Participantes do estudo}

Foi realizado um estudo observacional, analítico e transversal, envolvendo 18 pacientes com TAB, diagnosticados por avaliação clínica, e 15 indivíduos do grupo controle. Os pacientes foram selecionados na rotina de atendimento no ambulatório de transtornos de humor (ATHUA), do Hospital São Vicente de Paulo (HSVP) da Secretaria de Saúde do Distrito Federal, e os indivíduos do grupo controle foram selecionados na comunidade local, entre dezembro de 2019 e junho de 2020.

Foram incluídos pacientes com diagnóstico de TAB de acordo com o Manual Diagnóstico e Estatístico de Transtornos Mentais 5. a edição (DSM-5) em acompanhamento clínico regular, com idade de 18 a 60 anos. Foram excluídos pacientes com outras comorbidades psiquiátricas ou condições neurológicas que possam afetar o desempenho neurocognitivo; qualquer história de uso abusivo ou dependência de substâncias psicoativas ou álcool. Já para o grupo controle foram selecionados indivíduos com as características demográficas semelhantes aos pacientes, sem histórico de doenças psiquiátricas e uso de medicamentos psicotrópicos, bem como dependência química prévia ou atual.

Todos os participantes incluídos foram submetidos à avaliação dos parâmetros cognitivos por meio da aplicação da Escala COBRA durante entrevista médica. Além disso, outros dados clínico-demográficos foram coletados, tais como idade, sexo, escolaridade e estado civil. Nos pacientes com TAB, também foram coletadas informações a respeito da situação ocupacional, tratamento farmacológico, número de internações, idade e tempo de diagnóstico e história familiar de transtornos neuropsiquiátricos.

O presente estudo foi aprovado pelo Comitê de Ética em Pesquisa da Fundação de Ensino e Pesquisa em Ciências da Saúde (FEPECS), número do CAAE: 19359219.5.0000.5553. Aos indivíduos selecionados como participantes foi feito o esclarecimento da pesquisa e aqueles que concordaram em participar assinaram 0 Termo de Consentimento Livre e Esclarecido.

\section{Avaliação cognitiva pela escala COBRA}

A COBRA é uma escala de autorrelato que permite aferir dificuldades cognitivas de caráter subjetivo. Este instrumento é composto por 16 questões, as quais abordam se o indivíduo tem problemas para se lembrar do nome de pessoas, de acontecimentos passados importantes ou de leituras e conversas recentes, assim como em se orientar no tempo e espaço. Há também perguntas que visam mensurar dificuldades relacionadas a encontrar objetos de uso diário, manter a concentração em atividades, finalizar uma tarefa iniciada, aprender novas informações, realizar cálculos simples, entre outras. Em suma, esta escala avalia queixas cognitivas relacionadas à função executiva, velocidade de processamento, memória de trabalho, aprendizagem e memória verbal, e atenção/concentração (ROSA AR, et al., 2013).

Para cada item, a frequência é avaliada em uma escala de quatro pontos, atribuídos conforme a seguir: 0 = nunca; 1 = às vezes; 2 = frequentemente e 3 = sempre. Quanto mais elevada a pontuação, considera-se pior o desempenho cognitivo. Neste estudo, foi empregada a escala traduzida para o português e validada por (LIMA FM, et al., 2018), utilizando-se o valor de 10 como ponto de corte para disfunção cognitiva.

\section{Análise Estatística}

Associações entre as variáveis qualitativas foram avaliadas pelo teste Qui-Quadrado de Pearson e expressas em frequência absoluta (n) e relativa (\%). Já as variáveis quantitativas foram submetidas ao teste de normalidade de Shapiro-Wilk. Aquelas que apresentaram distribuição normal foram expressas em média e desvio padrão, e as que apresentaram distribuição não normal, em mediana e intervalo interquartil (Q3 Q1). Comparações entre os dois grupos (pacientes com TAB e controles) foram realizadas pelo teste $t$ de Student ou de Mann-Whitney (para valores com distribuição normal ou não normal, respectivamente). Além 
disso, foram avaliadas correlações entre as variáveis numéricas pelo teste de correlação de Pearson ou de Spearman (para variáveis com distribuição normal ou não normal, respectivamente). Todos os testes estatísticos foram bilaterais e considerou-se estatisticamente significativos os valores de $p<0,05$.

\section{RESULTADOS}

As características clínico-demográficas dos participantes do estudo estão apresentadas na Tabela 1. A maioria dos pacientes era do sexo feminino $(77,8 \%)$, com mediana de idade de 39 anos, e de escolaridade de 11 anos. Os controles, por sua vez, apresentaram características demográficas semelhantes $(73,3 \%$ do sexo feminino, com mediana de idade de 40 anos, e de escolaridade de 11 anos). A média de IMC dos participantes também foi aproximada, sendo $29,8 \pm 5,5 \mathrm{~kg} / \mathrm{m}^{2}$ nos pacientes e $28,4 \pm 5,7 \mathrm{~kg} / \mathrm{m}^{2}$ nos controles.

Por outro lado, a maioria dos pacientes $(55,6 \%)$ relatou a presença de transtorno neuropsiquiátrico na família, o que não foi observado nos controles. Ainda, dentre os pacientes, 33,3\% relataram histórico familiar de TAB. A média de idade ao diagnóstico foi de $28,5 \pm 5,5$ anos, enquanto do tempo de diagnóstico foi de $11,1 \pm 8$ anos e do número de internações de $3,8 \pm 3,3$ vezes. Em relação à situação ocupacional, apenas quatro pacientes $(22,2 \%)$ reportaram estar trabalhando atualmente, e a média de afastamento de trabalho foi de $5,7 \pm 2,4$ anos.

Tabela 1 - Características clínico-demográficas dos participantes do estudo ( $n=33)$. Brasília - DF, 2020.

\begin{tabular}{|c|c|c|c|}
\hline Variáveis & $\begin{array}{c}\text { TAB } \\
(n=18)\end{array}$ & $\begin{array}{c}\text { Controle } \\
(n=15)\end{array}$ & $p$ \\
\hline \multicolumn{4}{|l|}{ Sexo, n (\%) } \\
\hline Feminino & $14(77,8 \%)$ & $11(73,3 \%)$ & \multirow{2}{*}{$1,000^{\mathrm{a}}$} \\
\hline Masculino & $4(22,2 \%)$ & $4(26,7 \%)$ & \\
\hline Idade em anos, mediana (IIQ) & $39(17,25)$ & $40(20)$ & $0,901^{\mathrm{b}}$ \\
\hline Escolaridade em anos, mediana (IIQ) & $11(3)$ & $11(7)$ & $0,873^{b}$ \\
\hline Tabagista, n (\%) & $2(11,1 \%)$ & $0(0 \%)$ & $0,183^{\mathbf{a}}$ \\
\hline IMC $\left(\mathrm{kg} / \mathrm{m}^{2}\right)$, média $\pm \mathrm{DP}$ & $29,8 \pm 5,5$ & $28,4 \pm 5,7$ & $0,481^{c}$ \\
\hline COBRA, média \pm DP & $20,6 \pm 9,7$ & $5,3 \pm 2,3$ & \multirow{3}{*}{$<0,001^{\mathrm{c}}$} \\
\hline Até 10 & $3(16,7 \%)$ & $15(100 \%)$ & \\
\hline$>10$ & $15(83,3 \%)$ & $0(0 \%)$ & \\
\hline História familiar de transtorno neuropsiquiátrico, n (\%) & $10(55,6 \%)$ & $2(13,3 \%)$ & $0,027^{a}$ \\
\hline História familiar de TAB, N (\%) & $6(33,3 \%)$ & & \\
\hline Idade (anos) ao diagnóstico, média \pm DP & $28,5 \pm 5,5$ & & \\
\hline Tempo de diagnóstico (anos), média \pm DP & $11,1 \pm 8$ & & \\
\hline Número de internações, média \pm DP & $3,8 \pm 3,3$ & & \\
\hline Trabalhando atualmente, n (\%) & $4(22,2 \%)$ & & \\
\hline Anos de afastamento, média \pm DP & $5,7 \pm 2,4$ & & \\
\hline \multicolumn{4}{|l|}{ Uso de psicotrópicos, $n$ (\%) } \\
\hline Estabilizadores de humor & $6(33,3 \%)$ & & \\
\hline Antipsicótico & $1(5,6 \%)$ & & \\
\hline Estabilizadores de humor + antipsicóticos & $11(61,1 \%)$ & & \\
\hline
\end{tabular}

Legenda: ${ }^{\top} T$ Teste Qui-Quadrado de Pearson (valores expressos em frequência absoluta e relativa); bteste de Mann-Whitney (valores expressos em mediana e intervalo interquartil: Q3 - Q1); 'Teste $t$ de Student (valores expressos em média \pm desvio padrão). COBRA = Escala de disfunções cognitivas no transtorno bipolar; DP = desvio padrão; IIQ = intervalo interquartil; IMC = índice de massa corporal; $\mathrm{TAB}$ = transtorno afetivo bipolar. Fonte: Moura MT, et al., 2021. 
No que se refere à avaliação cognitiva, pacientes com TAB apresentaram pior desempenho em comparação aos controles, o que foi verificado pela média de pontuação na COBRA significativamente maior (Tabela 1, Figura 1). Além disso, quinze pacientes (83,3\%) apresentaram pontuação nesta escala maior que 10.
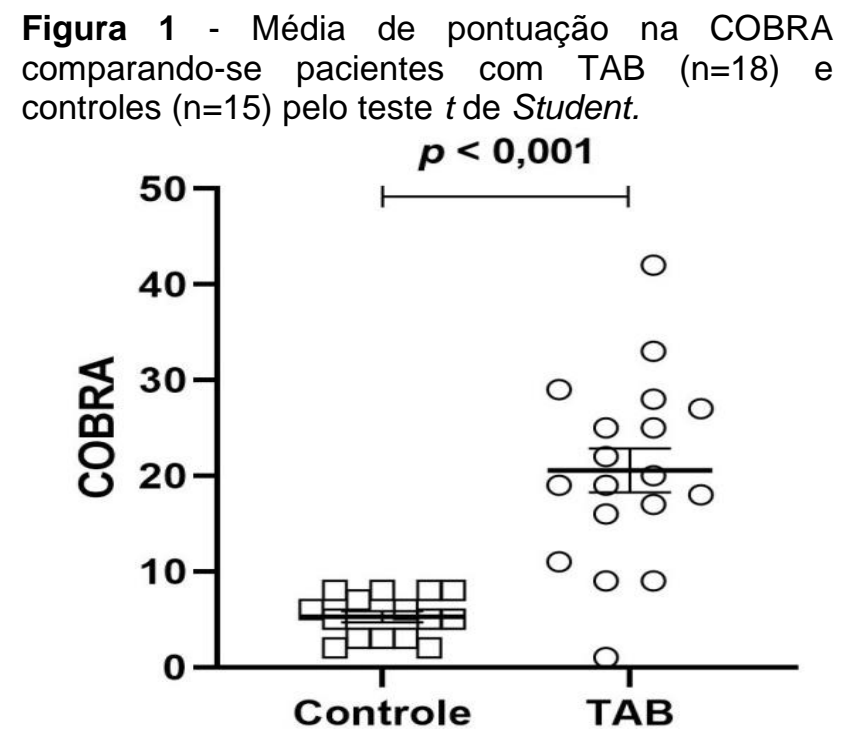

Fonte: Moura MT, et al., 2021

Adicionalmente, ao comparar o desempenho cognitivo dentre todos os pacientes em relação ao uso de antipsicóticos (típicos e atípicos), não foram encontradas diferenças significativas (Figura 2A). No entanto, comparando-se apenas os pacientes que pontuaram acima de 10 na escala, observou-se que as queixas cognitivas foram maiores naqueles que estavam em terapia com antipsicóticos, evidenciada pela pontuação significativamente maior (Figura 2B).

Figura 2 - Média de pontuação na COBRA comparando-se: A) todos os pacientes com TAB em uso ( $\mathrm{n}=12$ ) ou não ( $n=6)$ de antipsicóticos e; B) pacientes com TAB que pontuaram acima de 10 na COBRA em terapia com antipsicóticos $(n=10)$ ou não $(n=5)$, pelo teste $t$ de Student.

A

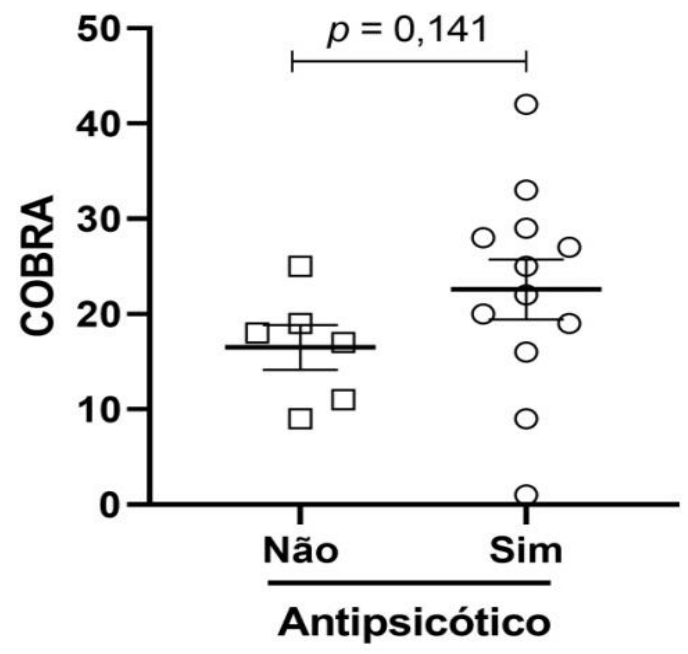

Todos os pacientes $(n=18)$
B

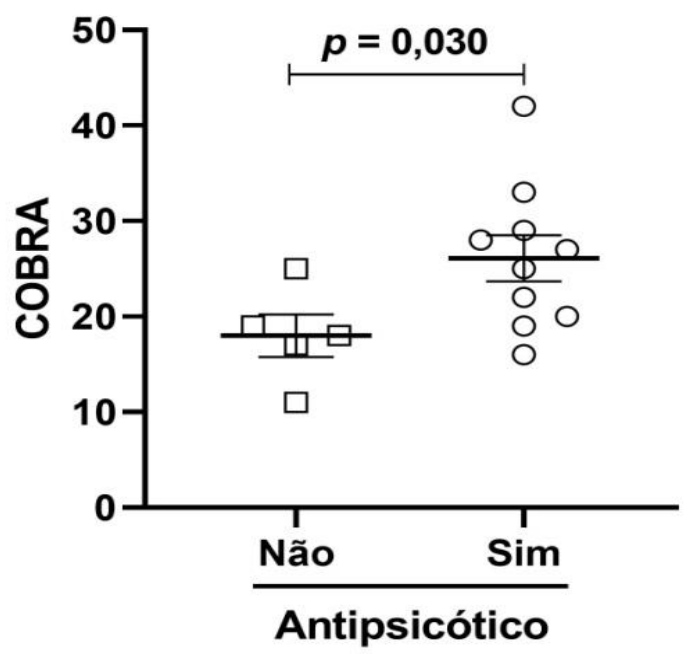

Pacientes com COBRA $>10(n=15)$

Fonte: Moura MT, et al., 2021. 
Por fim, o desempenho neurocognitivo não demonstrou correlação significativa com a idade ao diagnóstico ( $r$ de Pearson $=-0,098, p=0,700)$, o tempo de doença ( $r$ de Pearson $=0,341, p=0,166$ ), e o número de internações ( $r$ de Pearson $=0,184, p=0,465$ ). Ademais, a função cognitiva avaliada pela COBRA não foi correlacionada ao tempo de escolaridade, tanto no grupo de pacientes com TAB (rô de Spearman $=-0,083$, $p=0,744$ ), como no grupo controle (rô de Spearman $=-0,450, p=0,093$ ).

\section{DISCUSSÃO}

O resultado encontrado demonstra uma diferença de desempenho na escala COBRA entre os pacientes com TAB e o grupo controle. Essa observação se revela pela pontuação significativamente maior nos pacientes, com $83,3 \%$ pontuando acima de 10 . Assim, nesta amostra, foi possível evidenciar o prejuízo cognitivo decorrente da doença, ressaltando a importância da avaliação breve, que pode impactar na adesão do paciente ao tratamento, na necessidade de exames complementares, como a testagem neuropsicológica e nas estratégias farmacológicas (MISKOWIAK KW, et al., 2018).

De forma complementar, ao comparar o desempenho cognitivo dentre os pacientes em relação ao uso de antipsicóticos, não foram observadas diferenças significativas. No entanto, considerando apenas aqueles que pontuaram acima de 10 na escala, observou-se que os pacientes em uso de antipsicóticos apresentaram pior desempenho, evidenciado pela pontuação na COBRA significativamente maior em comparação àqueles que não estavam em uso destes medicamentos. Foi descrito previamente que a utilização de terapia antipsicótica em pacientes com esquizofrenia pode estar associada ao desenvolvimento de déficits cognitivos devido a suas ações antidopaminérgicas e anticolinérgicas, em adição ao comprometimento cognitivo decorrente da própria doença (KASPER S e RESINGER E, 2003).

De forma semelhante, pacientes com TAB, tanto em uso de antipsicóticos típicos quanto atípicos, também demonstraram prejuízo na cognição, indicando a existência de uma relação dose-dependente (BALANZÁMARTíNEZ V, et al., 2010; TAKEUCHI H, 2015; TORRENT C, et al., 2011). Diante disso, no consenso publicado por Miskowiak KW, et al. (2018) sugeriu-se a redução das doses de antipsicóticos, quando pertinente, para melhora do comprometimento cognitivo em pacientes com TAB.

A idade média ao diagnóstico foi de 28,5 anos, alguns anos após o início dos sintomas, que geralmente se dá entre o final da adolescência e início da vida adulta (BALDESSARINI RJ, et al., 2012). Esse dado condiz com a literatura, que aponta que o diagnóstico do TAB pode ocorrer de cinco a dez anos após o aparecimento dos sintomas (DAGANI J, et al., 2017). Esse tempo entre o início dos sintomas e o diagnóstico pode ocorrer por fatores como baixa assistência médica e erro diagnóstico, sendo que, com frequência, pacientes com TAB são inicialmente diagnosticados com depressão unipolar ou esquizofrenia (LUBLÓY Á, et al., 2020). Apesar de menos frequente, o TAB também pode ser diagnosticado na população infantil, sendo que, nesses casos, a confusão com outros transtornos psiquiátricos é ainda maior. Cabe ressaltar que a maior demora para início do tratamento associa-se a um pior prognóstico da doença (KAPCZINSKI F, et al., 2008).

Embora a perda cognitiva nos pacientes com TAB tenha sido associada à progressão da doença, neste trabalho não foram encontradas correlações entre idade ao diagnóstico, tempo de doença e número de internações com o resultado na COBRA, o que também foi observado em estudos anteriores (LIMA FM, et al., 2018; ROSA AR, et al., 2013).

Interessantemente, a pontuação na COBRA também não apresentou correlação significativa com o tempo de escolaridade, tanto no grupo de pacientes com TAB, como no grupo controle. Ainda que os resultados aqui apresentados não permitam inferir associação entre a escala e a situação educacional, vale-se ressaltar que a utilização da COBRA foi proposta para avaliação cognitiva em pacientes com TAB, como alternativa a outros instrumentos que são utilizados na rotina para avaliar o comprometimento cognitivo em síndromes demenciais. Destaca-se, dentre estes, o mini-exame do estado mental, considerado pouco sensível para avaliar déficits cognitivos em pacientes com transtornos psiquiátricos, em especial os mais jovens (ROSA AR, et al., 2013).

A escala COBRA, por sua vez, tampouco demonstrou-se adequada para detectar comprometimento cognitivo em pacientes com TAB com nível intelectual médio a alto (ROSA AR, et al., 2013). Apesar disso, em um estudo que subdividiu pacientes com TAB de acordo com o desempenho cognitivo avaliado pela 
COBRA, o grupo que apresentou cognição intacta possuía mais tempo de estudo em comparação àqueles que demonstraram maior prejuízo (LIMA F, et al., 2019).

$O$ relato da presença de transtornos neuropsiquiátricos em familiares de primeiro ou segundo grau foi significativamente maior em pacientes com TAB do que nos controles. Além disso, 33,3\% dos pacientes relataram a presença de $T A B$ na família, corroborando achados prévios que demonstraram grande carga genética para a doença (ÖZDEMIR O, et al., 2016; SMELAND OB, et al., 2020). Contudo, apesar de já ter sido relatada a influência do componente genético no $T A B$, estudos em famílias também já sugeriram que outras condições psiquiátricas podem fazer parte de um mesmo espectro fenotípico. Nesse sentido, diferentes transtornos psiquiátricos, como $\mathrm{TAB}$, depressão unipolar e esquizofrenia, poderiam ser manifestações distintas associadas a uma mesma predisposição genética (CARNEIRO FG e SORATTO MT, 2016).

Outro resultado que chamou a atenção no presente estudo foi o grande número de afastamentos entre os pacientes, com apenas quatro $(22,2 \%)$ atualmente trabalhando. Isso se reflete em um grande impacto social e econômico, conforme a Organização Mundial de Saúde (OMS), que considera a doença umas das principais causas de incapacitação do mundo (ALONSO J, et al., 2011). Considerando que os problemas na cognição estão relacionados ao comprometimento funcional, evidencia-se a importância do rastreio cognitivo nos pacientes com TAB (STREJILEVICH S, et al., 2013). Além de atingir majoritariamente indivíduos com faixa etária economicamente ativa, o TAB também acarreta elevados custos de saúde, uma vez que, muitas vezes, é necessário realizar a hospitalização do paciente durante as crises, as quais, também podem ser recorrentes (COSTA AMN, 2008).

Neste trabalho, os pacientes foram pareados com os controles por sexo, idade e escolaridade. Na amostra coletada, o número de pacientes do sexo feminino foi superior, apesar de, na população geral, o TAB ter distribuição igualitária entre os sexos (DIFLORIO A e JONES I, 2010). Isso pode ser atribuído ao perfil de pacientes que são atendidos no ambulatório, como também por haver uma maior tendência entre as mulheres na busca por tratamento psiquiátrico (MATHESON FI, et al., 2014). Fatores culturais, incluindo o preconceito com o transtorno mental e com o que se considera fragilidade masculina, são fortes contribuintes para que a procura por atendimento psiquiátrico seja menor entre os homens (CHATMON BN, 2020).

Este estudo apresenta algumas limitações: primeiramente, não foram utilizadas escalas para avaliação de mania, hipomania e depressão, mas apenas a avaliação clínica como critério de eutimia. Além disso, a amostra foi coletada em um ambulatório de serviço terciário. Desta forma, deve-se ter cautela ao extrapolar os resultados para pacientes que se encontram em outros contextos. Por fim, trata-se de um estudo transversal com número limitado de pacientes. Estudos posteriores, com desenho longitudinal e maior número amostral, são necessários para validação destes resultados. Apesar das suas limitações, este estudo foi um dos primeiros a-demonstrar a aplicabilidade da escala COBRA na rotina ambulatorial.

\section{CONCLUSÃO}

A avaliação de queixas cognitivas em pacientes com TAB, apesar de sua relevância, ainda não é realizada no cotidiano. Neste estudo, demonstrou-se a viabilidade de aplicação da escala COBRA na rotina dos atendimentos ambulatoriais em pacientes com esta condição psiquiátrica, a fim de se mensurar dificuldades subjetivas relacionadas à cognição. Com base nisso, é possível considerar que a incorporação deste instrumento na prática clínica pode qualificar o acompanhamento ambulatorial, minimizando prejuízos sociais e ocupacionais causados pelas dificuldades cognitivas apresentadas por esta população.

\section{REFERÊNCIAS}

1. ALONSO J, et al. Days out of role due to common physical and mental conditions: results from the WHO World Mental Health surveys. Molecular psychiatry, 2011; 16(12): 1234-1246.

2. ALTSHULER LL, et al. Neurocognitive function in clinically stable men with bipolar I disorder or schizophrenia and normal control subjects. Biological psychiatry, 2004; 56(8): 560-569.

3. AMERICAN PSYCHIATRIC ASSOCIATION. Diagnostic and statistical manual of mental disorders: DSM-5. 5th ed. Washington, D.C: 2013.

4. BALANZÁ-MARTÍNEZ V, et al. Neurocognition in bipolar disorders-a closer look at comorbidities and medications. European Journal of Pharmacology, 2010; 626(1): 87-96. 
5. BALDESSARINI RJ, et al. Age at onset versus family history and clinical outcomes in 1,665 international bipolar-I disorder patients. World Psychiatry, 2012; 11(1): 40-46.

6. BORTOLATO B, et al. Cognitive dysfunction in bipolar disorder and schizophrenia: a systematic review of metaanalyses. Neuropsychiatric disease and treatment, 2015; 11:3111.

7. BRASIL. Ministério da Saúde. Secretaria de Atenção à Saúde. Portaria № 315, de 30 de março de 2016. Aprova o Protocolo Clínico e Diretrizes Terapêuticas do Transtorno Afetivo Bipolar do tipo I, 2016.

8. CARNEIRO FG, SORATTO MT. Transtorno bipolar: fatores genéticos e ambientais. Enfermagem Brasil, 2016; 15(1): 39-43.

9. CARVALHO AF, et al. Bipolar disorder. New England Journal of Medicine, 2020; 383(1): 58-66.

10. CHATMON BN. Males and Mental Health Stigma. Am J Mens Health, 2020; 14(4): 1557988320949322.

11. COSTA AMN. Transtorno afetivo bipolar: carga da doença e custos relacionados. Archives of Clinical Psychiatry (São Paulo), 2008; 35(3): 104-110.

12. DAGANI J, et al. Meta-analysis of the interval between the onset and management of bipolar disorder. The Canadian Journal of Psychiatry, 2017; 62(4): 247-258.

13. DEPP CA, et al. Bipolar depression and cognitive impairment: shared mechanisms and new treatment avenues. Psychiatric Clinics, 2016; 39(1): 95-109.

14. DIFLORIO A; JONES I. Is sex important? Gender differences in bipolar disorder. International review of psychiatry, 2010; 22(5): 437-452.

15. GEDDES JR; MIKLOWITZ DJ. Treatment of bipolar disorder. The Lancet, 2013; 381(9878): 1672-1682.

16. GOODWIN FK; JAMISON KR. Manic-depressive illness: bipolar disorders and recurrent depression. Oxford University Press, 2007.

17. HILTY DM, et al. A review of bipolar disorder among adults. Psychiatric Services, 1999; 50(2): 201-213.

18. JAMES SL, et al. Global, regional, and national incidence, prevalence, and years lived with disability for 354 diseases and injuries for 195 countries and territories, 1990-2017: a systematic analysis for the Global Burden of Disease Study 2017. The Lancet, 2018; 392(10159): 1789-1858.

19. JENSEN JH, et al. Optimising screening for cognitive dysfunction in bipolar disorder: validation and evaluation of objective and subjective tools. Journal of affective disorders, 2015; 187: 10-19.

20. KAPCZINSKI F, et al. Allostatic load in bipolar disorder: implications for pathophysiology and treatment. Neuroscience \& Biobehavioral Reviews, 2008; 32(4): 675-692.

21. KASPER S; RESINGER E. Cognitive effects and antipsychotic treatment. Psychoneuroendocrinology, 2003; 28: 2738.

22. KURTZ MM; GERRATY RT. A meta-analytic investigation of neurocognitive deficits in bipolar illness: profile and effects of clinical state. Neuropsychology, 2009; 23(5): 551.

23. LIMA F, et al. Identifying cognitive subgroups in bipolar disorder: A cluster analysis. Journal of affective disorders, 2019; 246: 252-261.

24. LIMA FM, et al. Validity and reliability of the Cognitive Complaints in Bipolar Disorder Rating Assessment (COBRA) in Brazilian bipolar patients. Trends in psychiatry and psychotherapy, 2018; 40(2): 170-178.

25. LUBLÓY Á, et al. Exploring factors of diagnostic delay for patients with bipolar disorder: a population-based cohort study. BMC psychiatry, 2020; 20(1): 1-17.

26. MANN-WROBEL MC, et al. Meta-analysis of neuropsychological functioning in euthymic bipolar disorder: an update and investigation of moderator variables. Bipolar disorders, 2011; 13(4): 334-342.

27. MATHESON FI, et al. Physical health and gender as risk factors for usage of services for mental illness. J Epidemiol Community Health, 2014; 68(10): 971-978.

28. MISKOWIAK KW, et al. Assessing and addressing cognitive impairment in bipolar disorder: the International Society for Bipolar Disorders Targeting Cognition Task Force recommendations for clinicians. Bipolar disorders, 2018; 20(3): 184-194.

29. ÖZDEMIR O, et al. Family history in patients with bipolar disorder. Archives of Neuropsychiatry, 2016; 53(3): 276.

30. ROSA AR, et al. Validity and reliability of a rating scale on subjective cognitive deficits in bipolar disorder (COBRA). Journal of Affective Disorders, 2013; 150(1): 29-36.

31. SADOCK BJ, et al. Compêndio de Psiquiatria-: Ciência do Comportamento e Psiquiatria Clínica. Artmed Editora, 2016.

32. SHASTRY BS. Bipolar disorder: an update. Neurochemistry International, 2005; 46(4): 273-279.

33. SMELAND OB, et al. Genome-wide analysis reveals extensive genetic overlap between schizophrenia, bipolar disorder, and intelligence. Molecular psychiatry, 2020; 25(4): 844-853.

34. SQUARCINA L, et al. Twin studies for the investigation of the relationships between genetic factors and brain abnormalities in bipolar disorder. Epidemiology and psychiatric sciences, 2016; 25(6): 515-520.

35. STREJILEVICH S, et al. Mood instability and functional recovery in bipolar disorders. Acta Psychiatrica Scandinavica, 2013; 128(3): 194-202.

36. TAKEUCHI H. The Effect of Antipsychotic Dose-reduction On Cognition. European Psychiatry, 2015; 30(S1): 1-1.

37. TORRENT C, et al. Cognitive impairment in bipolar II disorder. The British Journal of Psychiatry, 2006; 189(3): 254259.

38. TORRENT C, et al. Effects of atypical antipsychotics on neurocognition in euthymic bipolar patients. Comprehensive psychiatry, 2011; 52(6): 613-622.

39. VIGO D, et al. Estimating the true global burden of mental illness. The Lancet Psychiatry, 2016; 3(2): 171-178.

40. YATHAM LN, et al. Canadian Network for Mood and Anxiety Treatments (CANMAT) and International Society for Bipolar Disorders (ISBD) 2018 guidelines for the management of patients with bipolar disorder. Bipolar disorders, 2018; 20(2): $97-170$. 